\title{
Research of algorithm for detecting suspected cheating behavior in examination surveillance video
}

\author{
Qi Xinda ${ }^{1, a}$ \\ ${ }^{1}$ Changchun Institute of Optics, Fine Mechanics and Physics, Chinese Academy of Science, \\ Changchun, China \\ aemail:qixinda@live.com
}

Keywords: Examination surveillance, video detection, intelligent processing

\begin{abstract}
It is difficult to review vast amounts of examination surveillance videos recently. In this paper, an algorithm for detecting suspected cheating behavior in examination surveillance video is proposed to solve this problem. The algorithm is based on adjacent frame difference algorithm, optical flow method and background difference method and optimizes them to detect suspected cheating from original examination surveillance videos. Then, the censors review the detected videos which contain the suspected cheating behavior and determine whether there is any cheating directly. This process is equivalent to review the original video review, thus the workload of reviewing original examination surveillance videos is reduced.
\end{abstract}

\section{Introduction}

Recently, there are more and more examinations which include more than 20 kinds of degree examination, about 23 foreign language examinations and lots of others in our country. At the same time, the number of annual examination is difficult to be statistically undetectable, as a result that a large number of examination surveillance video appear. The relevant departments need to review the videos and determine whether there is any cheating to guarantee the quality of the exam and fairness.

This process brings staggering workload. In jilin province college entrance examination, examination surveillance videos are more than 41540 hours. Tt takes 1730 days to review them based on the original method of review the original video, which brings a problem called "difficult review".

It is difficult to review vast amounts of examination surveillance videos recently. In this paper, an algorithm for detecting suspected cheating in examination surveillance video is proposed to solve this problem. The algorithm is divided into seven steps one by one. In the first step, the color video is transformed to gray image frame by frame. In the second step, we deal with the noise of image based on related algorithm. In the third step, we capture and update the image background. In the fourth step, background difference is obtained. In the fifth step, binaryzation is finished for images. In the sixth step, the regions which contain cheating are identified in the original videos. In the seventh step, the video clips which contain cheating are screened out and new video files are formed. Then, the censors review the detected videos which contain the suspected cheating and determine whether there is any cheating directly. This process is equivalent to review the original video review, thus the workload of reviewing original examination surveillance videos is reduced greatly.

\section{Algorithm principle}

At present, the commonly used and mature algorithm for moving target detection is background difference method ${ }^{[4]}$. In this method, firstly background is modeled to draw the background model $b_{k}(\mathrm{x}, \mathrm{y})$, and then the difference of the image $f_{k}(\mathrm{x}, \mathrm{y})$ and the background model $b_{k}(\mathrm{x}, \mathrm{y})$ is calculated to detect moving objects. In this paper, we will detect the cheating behavior from the examination surveillance video based on above algorithm. Most of the scenes are basically static in the examination surveillance video and only obvious cheating behavior can be determined, so we only need to detect moving targets that have large amplitude in the videos ${ }^{[1]}$. The detecting cheating behavior process is shown in figure 1, and the specific algorithm is described as follows: 


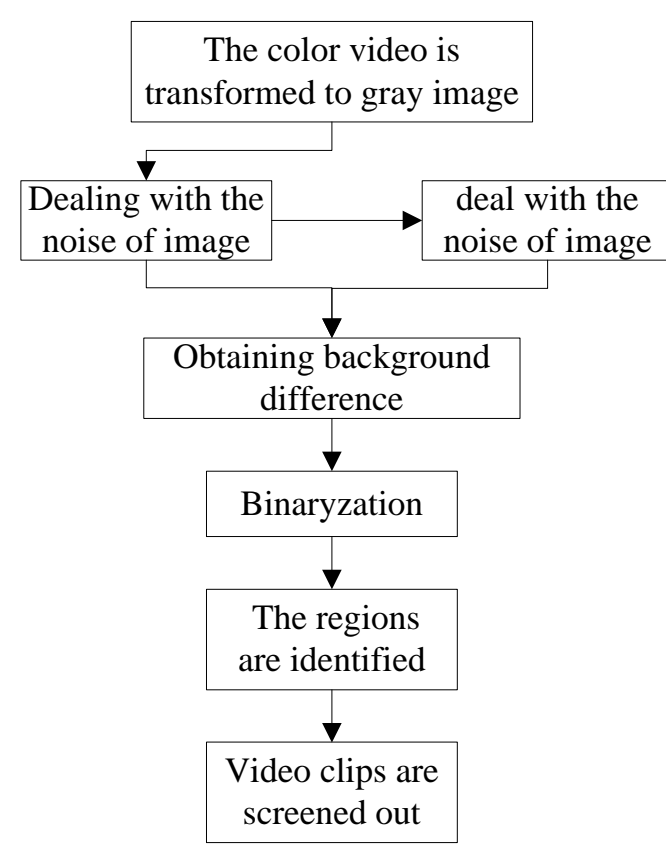

Fig. 1. Algorithm
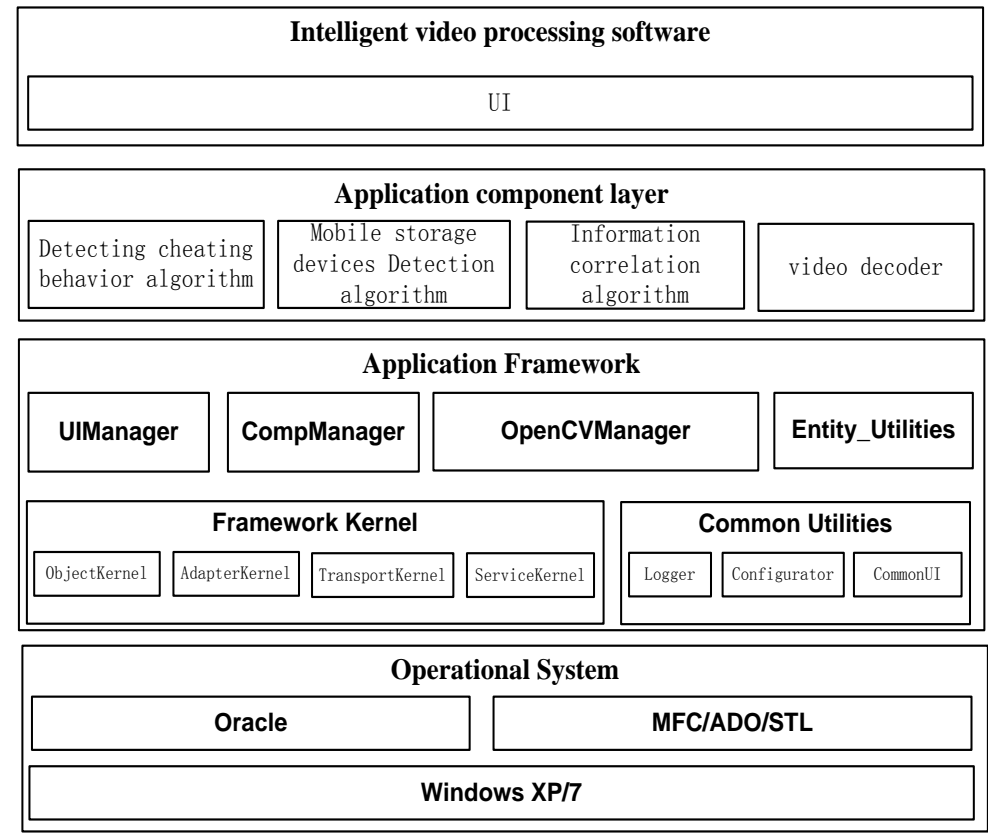

Fig. 2. Intelligent video processing software framework

(1). Converting color video to gray image

At present, the surveillance video is usually the color image, but if we have the gray information of the image, the cheating behavior can be detected. Therefore, the first step of the detection method of cheating is to convert the source video to gray image ${ }^{[2]}$. In the actual process, we convert color video to gray image based on the formula 1 . In the formula 1 , Gray denotes the gray value of pixels, $R$, G, B respectively says the red component green component or blue component. 0.30, 0.59, 0.11 which are the best value in experimental and theoretical derivation respectively is the best gray-scale image weights.

$$
\text { Gray }=0.30 \times R+0.59 \times G+0.11 \times B
$$

(2). Dealing with the noise of image

In the process of eliminating noise, on the image area noise processing [3] by using the method of median filter in the spatial domain, in the frequency domain using the method of low-pass filter to remove noise. In the spatial domain, we adopt the method of median filter to process image noise. In frequency domain, we use the method of low-pass filter to remove noise. Because the noise the method used in this paper are well known, this is no longer a detailed description..

(3). Capturing and updating the image background

After the analysis, during the process of capturing and updating the image background we firstly superimpose $\mathrm{N}$ image, and get the average value, and then updated.

(4). Obtaining background difference

The background difference method is also called the background subtraction method ${ }^{[5]}$, In this method, firstly background is modeled to draw the background model $b_{k}(\mathrm{x}, \mathrm{y})$, and then The difference of the image $f_{k}(\mathrm{x}, \mathrm{y})$ and the background model $b_{k}(\mathrm{x}, \mathrm{y})$ is calculated to detect moving objects. Before this process, we should rationally dividing the pixel based on inter frame, and meet prerequisite which means that the background changes little or slow. As similar as the continuous frame difference method, we need to complete binaryzation of the difference image $D_{K}(\mathrm{x}, \mathrm{y})$ with T as the threshold, and then draw the image binaryzation value. Differential formula is formula 2.

$$
D_{K}(\mathrm{x}, \mathrm{y})=\left|f_{k}(\mathrm{x}, \mathrm{y})-b_{k}(\mathrm{x}, \mathrm{y})\right|
$$




$$
R_{K}= \begin{cases}1 & \text { Foreground pixel } D_{K}(\mathrm{x}, \mathrm{y})>\mathrm{T} \\ 0 & \text { Background pixel } D_{K}(\mathrm{x}, \mathrm{y}) \leq \mathrm{T}\end{cases}
$$

(5). Binaryzation

We finish the process of the image binaryzation according to the formula $3^{[6]}$.

(6). Identifying the regions which contain cheating behavior

After we detect moving objects based on the background differencing, we obtain the maximum length of moving targets in $\mathrm{x}$ and $\mathrm{y}$ direction. Then the maximum length in $\mathrm{x}$ and $\mathrm{y}$ direction is respectively defined as the length and width of the rectangular that is marked in red and used to identify a region with cheating behavior the corresponding frame used in the red rectangle identifies with the area of cheating In the corresponding frame.

(7). Screening the video clips

We firstly get the time when the cheating appears, then find out the frame in the time, synthesize these frames for video clips synthesize these frames for video clips according to the time sequence in original video.

\section{Algorithm implementation}

The algorithm for detecting suspected cheating behavior in examination surveillance video is implemented in intelligence video processing software. This software selects the video clips which contains cheating behavior from the original videos automatically based on OpenCV ${ }^{[7]}$ that Integrates the adjacent frame difference algorithm, optical flow method, background difference method, the Adaboost algorithm. Then, the censors review the detected videos which contain the suspected cheating and determine whether there is any cheating directly. This process is equivalent to review the original video review, thus the workload of reviewing original examination surveillance videos is reduced greatly. The intelligence video processing software architecture is shown in figure 2 .

As shown in figure 2, the software architecture has is four layers based on components. The four layers are system software, application framework, application component and interface. The design of software architecture also can be explained from two angles which are the infrastructure and extensible architecture. System software and application framework are infrastructures of the intelligence video processing software, and application component and interface are extensible architecture. The software architecture has good flexibility for Software updating and upgrading.in the future.

Application component layer includes detecting cheating behavior algorithm component, mobile storage devices component, information correlation algorithm component and video decoder. This layer is the core layer in which the algorithm for detecting suspected cheating behavior in examination surveillance video is implemented. Mobile storage devices component can help the censors quickly find the video file, so that they can quickly enter the working state. Other software layers are as shown in figure 3.

\section{Case study}

There is a case in which intelligence video processing software detected the suspect cheating behavior of a examination in the figure 3 . We can see that when the examinee in the examination stood up, the software prompted that there were abnormal behavior. In such cases, the software prompts information with the support of the algorithm, selects the video clips, and forms a new video file. The censors review the detected videos which contain the suspected cheating and determine whether there is any cheating directly. 


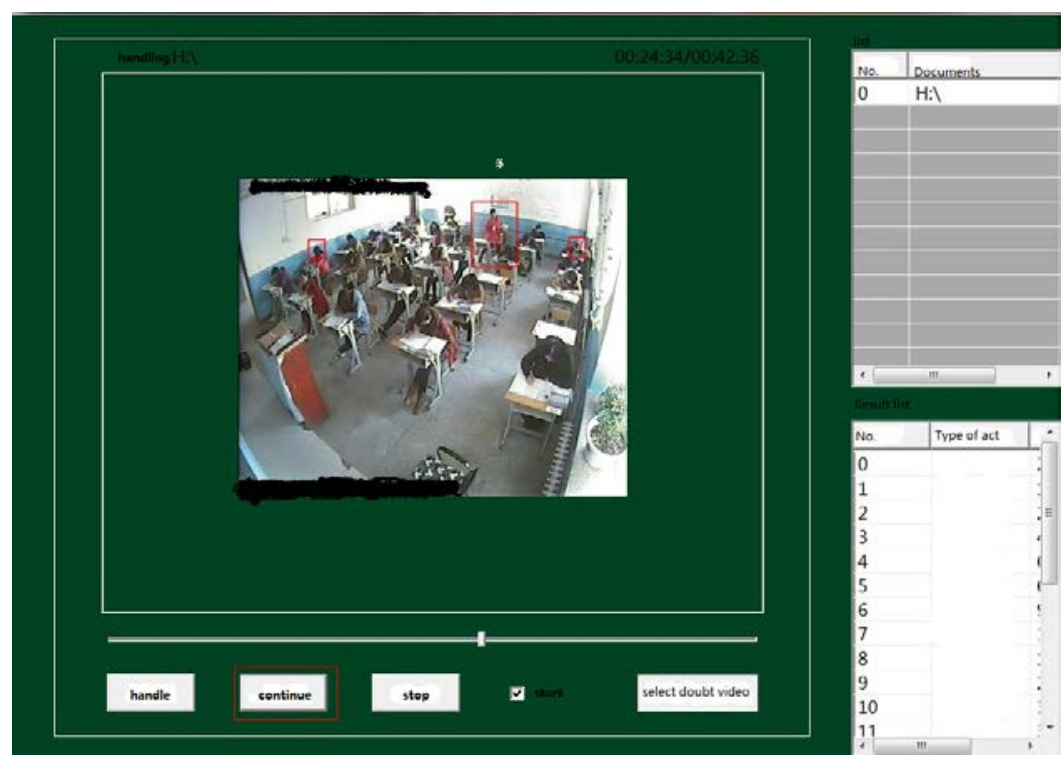

Fig.3. Intelligence video processing software running status

The software was used to detect the suspected cheating behavior in examination surveillance video of a self-study exam. The results showed that the time of the selected video clips was about $6 \%$ of the original video and the workload of video review was reduced more than $90 \%$.

\section{Conclusion}

In this paper, we proposed an algorithm for detecting suspected cheating behavior in examination surveillance video, and implemented it in the intelligence video processing software. The algorithm can detect suspected cheating from original examination surveillance videos. Then, the censors review the detected videos which contain the suspected cheating and determine whether there is any cheating directly. Under normal circumstances, the time of the selected video clips is less than $10 \%$ of the original video, which reduce the workload of reviewing examination surveillance video.

\section{References}

[1] R. I. Hartley.Theory and practice of projective rectifi cation [J]. International Journal of Computer Vision, 1998, 35:115-127.

[2] Rawat A.K., Singh J. Improved Median Filter using ROAD for Removal [J]. International Journal of Computer Applications, 2014, 7(35): 29-33.

[3] Chen, T. et al, (2001b). Space variant median filters for the restoration of im-pulse noise corrupted images [J]. IEEE Trans. Circuits Syst. II,Analog Digit. Signal Process., 2001, 8(48):784-789.

[4] Luo, W.. A new efficient impulse detection algorithm for the removal of im-pulse noise [J]. IEICE Trans. Fundam., 2005,10(E88-A), 2579-2586.

[5] Kokaram, A.C. et al (1995). Detection of missing data in image sequences [J]. IEEE Transaction on image processing, 2005, 11(4): 1496-1508.

[6] Srinivasan, K.S.. A New Fast and Efficient Decision-Based Algorithm for Re-moval of High-Density Impulse Noise [J] IEEE signal processing letter, 2007, 3(14):189-192.

[7] Barron J, Fleet D .Performance of optical flow techniques[J]. International Journal of Computer Vision, 1994, 12 (1):42-77. 